\title{
Monotonicity of power in games with a priori unions
}

\author{
J.M Alonso-Meijide ${ }^{1}$, C. Bowles ${ }^{2}$, M. J. Holler ${ }^{3}$, S. Napel ${ }^{4}$
}

May 8, 2006

No. 145

\begin{abstract}
Power indices are commonly required to assign at least as much power to a player endowed with voting weight $w$ than to any player of the same game with smaller weight. This local monotonicity and a related global property however are frequently and for good reasons violated when indices take account of a priori unions amongst subsets of players (reflecting, e.g., ideological proximity). This paper introduces adaptations of the conventional monotonicity notions that are suitable for voting games with an exogenous coalition structure. Relationships between these new monotonicity concepts are investigated and different coalitional versions of the Banzhaf and Shapley-Shubik power indices are compared accordingly.
\end{abstract}

Keywords: monotonicity; voting power; coalitional values; coalition structures; a priori unions AMS classification: $91 \mathrm{~A} 12,91 \mathrm{~A} 80$.

1 meijide@lugo.usc.es, Department of Statistics and Operations Research, University of Santiago de Compostela

2 carlos.bowles@ecb.int, Economic Department, European University Institute

${ }^{3}$ Corresponding author. holler@econ.uni-hamburg.de, Department of Economics, University of Hamburg

4 napel@econ.uni-hamburg.de, Department of Economics, University of Hamburg 


\section{Introduction}

Power indices measure players' abilities to influence outcomes in voting situations. They are valuable instruments to study power - arguably the most important concept in political sciences because the latter is generally not proportional to the voting weights at its source. On the one hand, this observation is obvious from considering, say, a $50 \%$ majority rule applied to an institution with three players and respective weights of (a) 51, 44, and 5 percent or (b) 49, 44, and 7 percent: Even though players 2 and 3 have a non-negligible share of voting weight in (a), they are in fact powerless. In contrast, all three players face a perfectly symmetric need (and opportunity) to find at least one coalition partner in order to pass a proposal in (b), i.e., they a priori have the same voting power. On the other hand, the actual translation of weights into power typically is not as straightforward as in (a) or (b), where it seems common sense to describe the situation by power vectors $(1,0,0)$ and $\left(\frac{1}{3}, \frac{1}{3}, \frac{1}{3}\right)$, respectively - at least if no further information about players' inclinations to form coalitions is available.

Several alternative indices have been proposed as suitable mappings from weights and decision quota to power. The most widely used ones are the Banzhaf and Shapley-Shubik power indices (Penrose, 1946, Banzhaf, 1965, Coleman, 1971; Shapley and Shubik, 1954), but there are many others. ${ }^{1}$ In determining which of all these indices is most suitable in a given context, the respective axiomatic characterizations and probabilistic foundations play an important role - in addition to the focus of this paper: monotonicity properties. For example, it is true for some but not all power indices that a player endowed with voting weight $w$ is for any voting game under consideration identified as at least as powerful than any player in the same game with smaller weight. This property is known as local monotonicity, and there is a similar property referring to cross-game comparisons called global monotonicity. Though this may not be obvious at first sight, there are good reasons why some indices are not monotonic in one, the other, or both senses (see Holler and Napel, 2004a and 2004b). This is true in particular when there exists information on the relations among players that is relevant for formation of a winning coalition (defined by jointly meeting the specified quota). Special relations among subsets of players can derive from their previous interaction, ideological proximity, geographic proximity, etc.

In this paper, we will specifically consider the case when information about players' inclinations to form coalitions is given by a partition of the set of players into pairwise disjoint a priori unions. This entails the assumption that either all members of any a priori union join a winning coalition, or none of them does. Games with such a coalition structure were first considered by Aumann and Drèze (1974), who extended the Shapley value to this new framework. A second approach was initiated by Owen (1977). Its main advantage is to allow for a transparent 'correction' of

\footnotetext{
${ }^{1}$ See Felsenthal and Machover (2006) for a historical survey.
} 
traditional power indices, while keeping track of their fundamental axiomatic properties with respect to the allocation of rewards and power both within and between unions. For example, Owen (1977) twice invokes the Shapley value, while Owen (1982) applies the Banzhaf value within and between unions. In contrast, Alonso-Meijide and Fiestras-Janeiro (2002) use the Banzhaf value in the game between unions and apply the Shapley value to surplus sharing within unions. Practical applications of values for games with a priori unions include Carreras and Owen (1988), Vázquez et al. (1997), and Alonso-Meijide and Bowles (2005). ${ }^{2}$

Since coalitional power indices typically do not obey the conventional notions of local and global monotonicity, the latter have naturally not played any role in discriminating between different coalitional power indices - let alone in their axiomatization. This is a main motivation for this paper. Its aim is to meaningfully extend local and global monotonicity to weighted majority games with a priori unions. This allows to illustrate the distinguishing features and behavior of coalitional power indices with and without reference to traditional indices.

The paper is organized as follows. In Section 2 we introduce our notation, formally state the conventional notions of local and global monotonicity, and give definitions of the most common power indices used for games with and without a given system of a priori unions. Then Sections 3 and 4 introduce two new notions of local monotonicity and two new notions of global monotonicity. We discuss their relationship and illustrate the new concepts by investigating the properties of three different coalitional power indices. Section 5 concludes.

\section{Preliminaries}

A finite (cooperative) game is a pair $(N, v)$ where $N=\{1,2, \ldots, n\}$ is the set of players and $v$, the characteristic function, is a real valued function defined on the subsets of $N$ (referred to as coalitions) such that $v(\varnothing)=0$. A simple game is a game $(N, v)$ in which $v$ only takes the values 0 and 1 , is not identically 0 , and satisfies the condition $v(T) \leq v(S)$ whenever $T \subseteq S$. A coalition $S$ is winning if $v(S)=1$, and losing if $v(S)=0$. For given $(N, v)$, the collection of all winning coalitions is referred to as $W$.

A simple game $(N, v)$ is a weighted majority game if there exists a set of weights $w_{1}, w_{2}, \ldots, w_{n}$ for the players, with $w_{i} \geq 0(i \in N)$ and $\sum_{i} w_{i}=1$, and a quota $q \in(0,1]$ such that $S \in W$ if and only if $w(S) \geq q$, where $w(S)=\sum_{i \in S} w_{i}{ }^{3}$ A weighted majority game is represented by

\footnotetext{
${ }^{2}$ For axiomatic characterizations see, e.g., Winter (1992), Vázquez et al. (1997), Amer et al. (2002), or Hamiache (1999). Procedures to compute coalitional values efficiently can be found in Owen and Winter (1992), Carreras and Magaña (1994), Alonso-Meijide et al. (2005), and Alonso-Meijide and Bowles (2005); the latter paper exploits generating functions while all others are based on a game's multilinear extension.

${ }^{3}$ See Taylor and Zwicker (1999) for a characterization of the simple games that are weighted majority games.
} 
$\left[q ; w_{1}, w_{2}, \ldots, w_{n}\right]$ and we denote the set of all weighted majority games with player set $N$ by $\mathcal{W}(N)$

A power index for weighted majority games is a function $g: \mathcal{W}(N) \rightarrow \mathbb{R}^{n}$ (or, more precisely, a family of functions because $N$ and $n$ are not fixed) which assigns to a weighted majority game $(N, v)$ a vector $g(N, v)$, where the real number $g_{i}(N, v)$ is the power of player $i$ in the game $(N, v)$ according to $g$.

The most important power indices are the Banzhaf index and the Shapley-Shubik index (hereafter BZ and SH index). These indices can be written ss $^{4}$

$$
g_{i}(N, v)=\sum_{S \subseteq N \backslash i} p_{S}^{i} \cdot[v(S \cup i)-v(S)], \text { for any } i \in N,
$$

where $\left\{p_{S}^{i}: S \subseteq N \backslash i\right\}$ corresponds to a probability distribution over the collection of coalitions not containing $i$. For the BZ index

$$
p_{S}^{i}=\frac{1}{2^{n-1}}
$$

and for the SH index

$$
p_{S}^{i}=\frac{s !(n-s-1) !}{n !},
$$

where $s$ refers to the cardinality of $S$.

The difference $v(S \cup i)-v(S)$ is called the marginal contribution of player $i$ to coalition $S$. Taking into account that for a simple game $v(T)=1$ if $T \in W$ and $v(T)=0$ otherwise, it holds that $v(S \cup i)-v(S)=1$ if and only if $S$ is losing and $S \cup i$ is winning. In this case, we say that the pair of coalitions $(S, S \cup i)$ is a swing for player $i$.

Given $(N, v) \in \mathcal{W}(N)$ two players $i, j \in N$ are called symmetric if their marginal contribution to any coalition is the same. A power index $g$ is symmetric if the power of two symmetric players is always the same.

An intuitively compelling property in the context of weighted majority games is local monotonicity:

Definition 1 A power index $g: \mathcal{W}(N) \rightarrow \mathbb{R}^{n}$ is locally monotonic if for each weighted majority game $(N, v)=\left[q ; w_{1}, w_{2}, \ldots, w_{n}\right] \in \mathcal{W}(N)$

$$
g_{i}(N, v) \geq g_{j}(N, v)
$$

holds for every pair of players $i, j \in N$ such that $w_{i}>w_{j}$.

Note that the marginal contribution of a player $i \in N$ to any coalition $S$ weakly increases in weight $w_{i}$ : if player $i$ with weight $w_{i}$ can turn a losing coalition into a winning one by joining, then any

\footnotetext{
${ }^{4}$ In line with the literature, we write $S \backslash i$ instead of $S \backslash\{i\}$ and $S \cup i$ instead of $S \cup\{i\}$. Also note that we consider the non-normalized version of the Banzhaf index.
} 
player $j$ with weight $w_{j}>w_{i}$ can necessarily do so, too. Moreover, the coefficients $p_{S}^{i}$ in Eq. (1) only depend on the cardinality of $N$ for the BZ index and on the cardinality of $S$ and $N$ for the SH index, i.e., they are constant in $w_{i}$. It follows that for given $\left[q ; w_{1}, w_{2}, \ldots, w_{n}\right]$ with $w_{i}>w_{j}$, the BZ (SH) index of player $i$ is at least as big as the $\mathrm{BZ}(\mathrm{SH})$ index of player $j$, i.e., both indices satisfy local monotonicity.

While local monotonicity refers to the relation between power of two players in the same game, another intuitively desirable property looks at power of the same player in two different weighted majority games:

Definition 2 A power index $g: \mathcal{W}(N) \rightarrow \mathbb{R}^{n}$ is globally monotonic if for every two weighted majority games $(N, v)=\left[q ; w_{1}, w_{2}, \ldots, w_{n}\right]$ and $\left(N, v^{\prime}\right)=\left[q ; w_{1}^{\prime}, w_{2}^{\prime}, \ldots, w_{n}^{\prime}\right] \in \mathcal{W}(N)$

$$
g_{i}(N, v) \geq g_{i}\left(N, v^{\prime}\right)
$$

holds for every player $i \in N$ such that $w_{i}>w_{i}^{\prime}$ and $w_{j} \leq w_{j}^{\prime}$ for all $j \neq i$.

Both BZ and SH indices satisfy global monotonicity (see Turnovec 1998). Turnovec (1998) proves, too, that any power index $g$ which is globally monotonic and symmetric, is also locally monotonic. So, loosely speaking, local monotonicity is a specialization of global monotonicity.

We will next formalize an exogenous division of players $N=\{1, \ldots, n\}$ into a priori unions which will either join a winning coalition completely or not at all. Denote by $\mathcal{P}(N)$ the set of all partitions of $N$. An element $P \in \mathcal{P}(N)$ is called a coalition structure or a system of unions of the set $N$. Two somewhat degenerate systems of unions are $P^{n}=\{\{1\},\{2\}, \ldots,\{n\}\}$ and $P^{N}=\{\{N\}\}$; all others introduce an asymmetry amongst the players which is generally unrelated to their voting weights. A weighted majority game with a coalition structure is a triplet $(N, v, P)$, where $(N, v) \in \mathcal{W}(N)$ and $P \in \mathcal{P}(N)$. The family of all weighted majority games with player set $N$ and a coalition structure is denoted by $\mathcal{W}^{\mathcal{P}}(N)$.

If $(N, v, P) \in \mathcal{W}^{\mathcal{P}}(N)$, with $P=\left\{P_{k}: k \in M, M=\{1, \ldots, m\}\right\}$, the quotient game $\left(M, v^{P}\right)$ induced by $(N, v, P)$ is the weighted majority game played between the unions, i.e.,

$$
\left(M, v^{P}\right) \in \mathcal{W}(M) \text { and } v^{P}(R)=v\left(\bigcup_{k \in R} P_{k}\right) \text { for all } R \subseteq M .
$$

The game $\left(M, v^{P}\right)$ can be represented by $\left[q ; w\left(P_{1}\right), w\left(P_{2}\right), \ldots, w\left(P_{m}\right)\right]$ where $w\left(P_{k}\right)=\sum_{i \in P_{k}} w_{i}$ with $k \in M, M=\{1, \ldots, m\}$.

As in the case without an explicit coalition structure, we call two players $i, j \in P_{k}$ symmetric in $(N, v, P) \in \mathcal{W}^{\mathcal{P}}(N)$ if their marginal contribution to every coalition is the same. Analogously, two a priori unions $P_{k}$ and $P_{l}$ are called symmetric if their marginal contribution to any coalition in the quotient game $\left(M, v^{P}\right)$ is the same.

As a straightforward extension of a power index defined on $\mathcal{W}(N)$, a (coalitional) power index for weighted majority games with a coalition structure is a function $g: \mathcal{W}^{\mathcal{P}}(N) \rightarrow \mathbb{R}^{n}$ which assigns 
to each game $(N, v, P)$ a vector $g(N, v, P)$, where the real number $g_{i}(N, v, P)$ is the power of player $i$ in the game according to $g$.

A coalitional power index $g$ is symmetric within unions if the power of two symmetric members of a union $P_{k}$ is the same. Intuitively, symmetry within unions guarantees that power differences for players in the same union must be based on differences in their weights (and not, e.g., different relations to other players inside or outside the union). Analogously, we say that $g$ is symmetric between unions if the sum of the individual power of players of two symmetric a priori unions is the same.

We will focus on three power indices defined on $\mathcal{W}^{\mathcal{P}}(N)$ : the Banzhaf-Owen index (hereafter BO index), the Symmetric Coalitional Banzhaf index (SCB index) and the Owen index (OW index). In analogy to Eq. (1), and letting player $i$ be contained in a priori union $P_{k}$, these indices can be written as

$$
g_{i}(N, v, P)=\sum_{R \subseteq M \backslash k} \sum_{T \subseteq P_{k} \backslash i} p_{R, T}^{i} \cdot\left[v\left(Q_{R} \cup T \cup i\right)-v\left(Q_{R} \cup T\right)\right] \text {, for any } i \in N,
$$

where $P=\left\{P_{1}, \ldots, P_{m}\right\}$ describes the coalition structure, $M=\{1, \ldots, m\}$ is $P$ 's index set, and $Q_{R}=\bigcup_{l \in R} P_{l}$ refers to the subset of players belonging to any union referred to by index subset $R \subseteq M$. The key difference to Eq. (1) is that a priori unions other than the $P_{k}$ which contains $i$ are assumed to either have joined completely (with all members) or not at all when the weighted average of $i$ 's marginal contributions is evaluated.

For the $\mathrm{BO}$ index the weights, usually interpreted as probabilities, are

$$
p_{R, T}^{i}=\frac{1}{2^{m-1}} \cdot \frac{1}{2^{p_{k}-1}},
$$

for the SCB index

$$
p_{R, T}^{i}=\frac{1}{2^{m-1}} \cdot \frac{t !\left(p_{k}-t-1\right) !}{p_{k} !}
$$

and for the OW index

$$
p_{R, T}^{i}=\frac{r !(m-r-1) !}{m !} \cdot \frac{t !\left(p_{k}-t-1\right) !}{p_{k} !}
$$

where $r, t$, and $p_{k}$ refer to the cardinality of sets $R, T$, and $P_{k}$, respectively (see Owen, 1982; Alonso-Meijide and Fiestras-Janeiro, 2002; and Owen, 1977, respectively).

It follows from these weights that one can view all three indices as describing a two-level decision making process: First, the respective $p_{k}$ members of the unions $P_{k} \in P$ take a decision amongst themselves - with influence on this decision measured using the probability model of either the SH or BZ index. Afterwards, (representatives of) the $m$ unions take an overall decision based on the respective bottom-level choices, where influence on the overall decision is again measured by either the $\mathrm{SH}$ or $\mathrm{BZ}$ index. Or one takes the perspective of the allocation of a surplus of transferable utility, corresponding to the worth of the grand coalition $v(N)=1$. Then one may interpret above 
probabilities as formalizing that unions or union representatives first split the total amount between the unions, and thereafter each union internally allocates its share. ${ }^{5}$ At each stage the respective opportunities for forming coalitions across and inside unions are taken into account in either the SH or BZ way. In particular for all games with coalition structures $P^{n}=\{\{1\},\{2\}, \ldots,\{n\}\}$ and $P^{N}=\{\{N\}\}$, which respectively imply true interaction only between or only within unions, the OW index coincides with the $\mathrm{SH}$ index of $(N, v)$, and the $\mathrm{BO}$ index coincides with the BZ index of $(N, v)$. The $\mathrm{SCB}$ index applies the $\mathrm{BZ}$ index to inter-union and the $\mathrm{SH}$ index to intra-union interaction, i.e., it coincides with the $\mathrm{BZ}$ index for $\left(N, v, P^{n}\right)$ and the $\mathrm{SH}$ index for $\left(N, v, P^{N}\right)$.

For illustration, consider the weighted majority game $(N, v)$ represented by $\left[\frac{68}{135} ; \frac{60}{135}, \frac{34}{135}, \frac{17}{135}, \frac{13}{135}, \frac{11}{135}\right]$ with coalition structure $P=\{\{1\},\{2,4,5\},\{3\}\}{ }^{6}$ Ignoring the information about the a priori union of players 2, 4 , and 5 , one can compute the $\mathrm{SH}$ and $\mathrm{BZ}$ power indices as

$$
g^{S H}(N, v)=\left(\frac{6}{10}, \frac{1}{10}, \frac{1}{10}, \frac{1}{10}, \frac{1}{10}\right)
$$

and

$$
g^{B Z}(N, v)=\left(\frac{7}{8}, \frac{1}{8}, \frac{1}{8}, \frac{1}{8}, \frac{1}{8}\right)
$$

Non-surprisingly, the respective power values are weakly increasing in voting weight, reflecting $g^{S H}$ 's and $g^{B Z}$ 's local monotonicity. In contrast, the OW, SCB, and BO coalitional power indices, which take coalition structure $P$ into account, yield

$$
\begin{aligned}
& g^{O W}(N, v, P)=\left(\frac{3}{9}, \frac{1}{9}, \frac{3}{9}, \frac{1}{9}, \frac{1}{9}\right), \\
& g^{S C B}(N, v, P)=\left(\frac{3}{6}, \frac{1}{6}, \frac{3}{6}, \frac{1}{6}, \frac{1}{6}\right),
\end{aligned}
$$

and

$$
g^{B O}(N, v, P)=\left(\frac{4}{8}, \frac{1}{8}, \frac{4}{8}, \frac{1}{8}, \frac{1}{8}\right)
$$

All clearly violate local monotonicity: $w_{2}>w_{3}$ but each of the coalitional indices indicates greater power for player 3 than for player 2 .

At an intuitive level, the a priori union between players 2, 4, and 5 means that feasible winning coalitions (or possible governments, in the political context that generated the example) never involve player 2 alone. So whatever spoils or influence on policy comes with belonging to a winning coalition - it has to be shared by player 2 with 4 and 5 . In contrast, player 3 (who is

\footnotetext{
${ }^{5}$ This interpretation implicitly assumes $\sum_{i \in N} g_{i}(N, v, P) \leq v(N)$, which does not generally hold if the (nonnormalized) BZ index is invoked within or between unions.

${ }^{6}$ This weighted majority game actually reflects the Catalonia Parliament, a typical Western Europe parliamentary body, during Legislature 1995-1999 (see Alonso-Meijide et al. 2005). From an abstract point of view it is an instance of the so-called apex game: the apex player 1 can form a minimal winning coalition with any other player and in addition only the coalition involving all small players is minimal winning (meaning that each member makes a positive marginal contribution).
} 
symmetric to player 2 ignoring $P$ ) only negotiates on its own behalf and by definition always is the unique swing player in its own bottom-level subgame. So player 2 keeps an undivided full share of spoils from the winning coalition potentially formed at the top level. Knowing the coalition structure formalized by $P$, we should thus expect the respective power numbers to violate local monotonicity: indices $g^{O W}, g^{S C B}$, and $g^{B O}$ would rather have a problem if they did not (cf. Holler and Napel, 2004a and 2004b). As we shall see, they satisfy different notions of monotonicity which are better suited to coalition structure $P$.

\section{Local monotonicity and coalition structures}

We will first consider restrictions of local monotonicity adapted to weighted majority games with a system of a priori unions. The subsequent section will then address global monotonicity, and relate both notions of monotonicity to each other. In either case, the most straightforward adaptation involves two separate monotonicity properties, within and between unions.

\subsection{Local monotonicity within unions}

Even when coalition formation in a given weighted majority game is restricted by a system of a priori unions, we would expect some kind of monotonicity at the 'very local' level, i.e., comparing players belonging to the same a priori union. This is naturally captured by

Definition 3 A coalitional power index $g: \mathcal{W}^{\mathcal{P}}(N) \rightarrow \mathbb{R}^{n}$ is locally monotonic within unions if for each weighted majority game with a coalition structure $(N, v, P) \in \mathcal{W}^{\mathcal{P}}(N)$, where $(N, v)=$ $\left[q ; w_{1}, w_{2}, \ldots, w_{n}\right] \in \mathcal{W}(N)$ and $P=\left\{P_{1}, P_{2}, \ldots, P_{m}\right\} \in \mathcal{P}(N)$,

$$
g_{i}(N, v, P) \geq g_{j}(N, v, P)
$$

holds for every pair of players $i, j \in P_{k}$ such that $w_{i}>w_{j}$ with $k \in M=\{1, \ldots, m\}$.

This property is weaker than conventional local monotonicity, ${ }^{7}$ because the weak inequality refers only to players $i$ and $j$ within the same union - not arbitrary pairs $i, j \in N$. If a power index satisfies local monotonicity then, of course, it also satisfies local monotonicity within unions. In turn, local monotonicity within unions only implies that general local monotonicity is not violated when the specific coalition structure $P^{N}=\{\{N\}\}$ is concerned (since in this degenerate case the inequality in fact refers to all players).

\footnotetext{
${ }^{7}$ Definition 1 refers to indices with domain $\mathcal{W}(N)$ and therefore, technically speaking, the two monotonicity notions cannot be compared. We here and later implicitly refer to the straightforward extension of local and global monotonicity to domain $\mathcal{W}^{\mathcal{P}}(N)$ (simply ignoring the given coalition structure).
} 
Again note that the marginal contribution of player $i \in N$ to a given coalition, corresponding to the difference $v\left(Q_{R} \cup T \cup i\right)-v\left(Q_{R} \cup T\right)$ in Eq. (2), weakly increases in weight $w_{i}$. The coefficients $p_{R, T}^{i}$ in Eq. (2) are constant in $w_{i}$ for all considered coalitional indices. We thus obtain

Proposition 1 The BO, SCB and $O W$ indices satisfy local monotonicity within unions.

\subsection{Local monotonicity between unions}

An additional and complementing notion of local monotonicity in games with a coalition structure refers to cross-union comparisons. It relates the aggregate weights of unions $P_{k}$ and $P_{l}$ to their aggregate power values, determined by the individual power values of the respective members. It is natural to use simple summation for the former aggregation and this is arguably the best way for the latter, too. We then say that a coalitional power index satisfies local monotonicity between unions if total power of a union cannot exceed total power of another union with greater total weight. Or, more formally:

Definition 4 A coalitional power index $g: \mathcal{W}^{\mathcal{P}}(N) \rightarrow \mathbb{R}^{n}$ is locally monotonic between unions if for each weighted majority game with a coalition structure $(N, v, P) \in \mathcal{W}^{\mathcal{P}}(N)$, where $(N, v)=$ $\left[q ; w_{1}, w_{2}, \ldots, w_{n}\right] \in \mathcal{W}(N)$ and $P=\left\{P_{1}, P_{2}, \ldots, P_{m}\right\} \in \mathcal{P}(N)$,

$$
\sum_{i \in P_{k}} g_{i}(N, v, P) \geq \sum_{i \in P_{l}} g_{i}(N, v, P)
$$

holds for every pair of a priori unions $P_{k}, P_{l} \in P$ such that $w\left(P_{k}\right)=\sum_{i \in P_{k}} w_{i}>w\left(P_{l}\right)=$ $\sum_{i \in P_{l}} w_{i}$.

Considering our earlier example $(N, v)=\left[\frac{68}{135} ; \frac{60}{135}, \frac{34}{135}, \frac{17}{135}, \frac{13}{135}, \frac{11}{135}\right]$ with $P=\{\{1\},\{2,4,5\},\{3\}\}$ again, we can see that the $\mathrm{BO}$ index violates local monotonicity between unions:

$$
w(\{2,4,5\})=\frac{58}{135}>w(\{3\})=\frac{17}{135},
$$

but

$$
\sum_{i \in P_{2}=\{2,4,5\}} g_{i}^{B O}(N, v, P)=\frac{3}{8}<\sum_{i \in P_{3}=\{3\}} g_{i}^{B O}(N, v, P)=\frac{4}{8} .
$$

In contrast, we obtain

$$
\sum_{i \in P_{2}=\{2,4,5\}} g_{i}^{O W}(N, v, P)=\sum_{i \in P_{3}=\{3\}} g_{i}^{O W}(N, v, P)=\frac{3}{6} .
$$

and

$$
\sum_{i \in P_{2}=\{2,4,5\}} g_{i}^{S C B}(N, v, P)=\sum_{i \in P_{3}=\{3\}} g_{i}^{S C B}(N, v, P)=\frac{4}{8} .
$$

for the OW and SCB indices, i.e., these are candidates for coalitional power indices satisfying local monotonicity between unions. 
A coalitional power index which is locally monotonic between unions does not violate general local monotonicity for games with the special coalition structure $P=P^{n}$ (since then all players form their own union). More generally, local monotonicity between unions amounts to conventional local monotonicity restricted to the quotient game $\left(M, v^{P}\right)$, which summarizes the interaction between unions. In fact, one can relate local monotonicity between unions of a coalitional power index $g$ to conventional local monotonicity of an underlying standard power index $f$ (if it exists), provided that $g$ treats the original game and its quotient game in a consistent fashion. In order to make this precise, we need to introduce two more concepts, namely the quotient game property (Winter, 1992) and coalitional extensions (Alonso-Meijide and Fiestras-Janeiro, 2002).

Definition 5 A power index $g: \mathcal{W}^{\mathcal{P}}(N) \rightarrow \mathbb{R}^{n}$ satisfies the quotient game property if for all $(N, v, P) \in \mathcal{W}^{\mathcal{P}}(N)$ and all $P_{k} \in P$

$$
\sum_{i \in P_{k}} g_{i}(N, v, P)=g_{k}\left(M, v^{P}, P^{m}\right),
$$

where $\left(M, v^{P}\right) \in \mathcal{W}(N)$ is the quotient game induced by $(N, v, P)$ and $P^{m}=\{\{1\}, \ldots,\{m\}\}$.

This requires that total power of any a priori union $P_{k}$ in $(N, v, P)$ is equivalent to the power of player $k$ (representative of union $P_{k}$ ) in the quotient game $v^{P}$ assuming the degenerate coalition structure $P^{m}=\{\{1\}, \ldots,\{m\}\}$.

The property is satisfied by the $\mathrm{OW}$ and $\mathrm{SCB}$ indices but not by the $\mathrm{BO}$ index (AlonsoMeijide and Fiestras-Janeiro, 2002). The latter can be seen for the five player unanimity game $(N, v)$ represented by $\left[1 ; \frac{1}{5}, \frac{1}{5}, \frac{1}{5}, \frac{1}{5}, \frac{1}{5}\right]$ with coalition structure $P=\{\{1,2,3\},\{4,5\}\}$. The BO index for this game is

$$
g^{B O}(N, v, P)=\left(\frac{1}{8}, \frac{1}{8}, \frac{1}{8}, \frac{2}{8}, \frac{2}{8}\right) .
$$

However, by applying coalition structure $P=\{\{1\},\{2\}\}$ in the quotient game $\left(M, v^{P}\right)$, represented by $\left[1 ; \frac{3}{5}, \frac{2}{5}\right]$, one obtains

$$
g^{B O}\left(M, v^{P}, P\right)=\left(\frac{1}{2}, \frac{1}{2}\right) .
$$

The notion that a coalitional power index $g$ derives from an underlying conventional power index can be made precise as follows:

Definition 6 Given a value $f: \mathcal{W}(N) \rightarrow \mathbb{R}^{n}$, a power index $g: \mathcal{W}^{\mathcal{P}}(N) \rightarrow \mathbb{R}^{n}$ is a coalitional extension of $f$ if

$$
g\left(N, v, P^{n}\right)=f(N, v)
$$

holds for every $(N, v) \in \mathcal{W}(N)$ with $P^{n}=\{\{1\}, \ldots,\{n\}\}$.

In particular, the OW index is a coalitional extension of the $\mathrm{SH}$ index, and the $\mathrm{BO}$ and $\mathrm{SCB}$ indices are coalitional extensions of the BZ index. Combining the notion of a coalitional extension with the quotient game property, we obtain: 
Proposition 2 If $g: \mathcal{W}^{\mathcal{P}}(N) \rightarrow \mathbb{R}^{n}$ is a coalitional extension of $f: \mathcal{W}(N) \rightarrow \mathbb{R}^{n}$ and satisfies the quotient game property, then local monotonicity of $f$ implies local monotonicity between unions of $g$.

Proof. Let $g$ be a coalitional extension of $f$ that satisfies the quotient game property and consider an arbitrary game $(N, v, P) \in \mathcal{W}^{\mathcal{P}}(N)$, where $(N, v)=\left[q ; w_{1}, w_{2}, \ldots, w_{n}\right] \in \mathcal{W}(N)$ and $P=$ $\left\{P_{k}: k \in M, M=\{1, \ldots, m\}\right\} \in \mathcal{P}(N)$. For each $P_{k} \in P$ we have

$$
\sum_{i \in P_{k}} g_{i}(N, v, P)=g_{k}\left(M, v^{P}, P^{m}\right)=f_{k}\left(M, v^{P}\right),
$$

where the first equality uses the quotient game property and the second that $g$ extends $f$. So

$$
\sum_{i \in P_{k}} g_{i}(N, v, P) \geq \sum_{i \in P_{l}} g_{i}(N, v, P)
$$

if and only if

$$
f_{k}\left(M, v^{P}\right) \geq f_{l}\left(M, v^{P}\right) .
$$

The latter and hence the former must be true for any pair of a priori unions $P_{k}, P_{l} \in P$ such that $w\left(P_{k}\right)=\sum_{i \in P_{k}} w_{i}>w\left(P_{l}\right)=\sum_{i \in P_{l}} w_{i}$ whenever $f$ is locally monotonic.

Corollary 1 The $S C B$ and $O W$ indices are locally monotonic between unions.

\section{Global monotonicity and coalition structures}

We now turn to global monotonicity, and show how above notions of local monotonicity within and between unions relate to their global analogues.

\subsection{Global monotonicity within unions}

Global monotonicity refers to the comparison of different games sharing a fixed set of players. The same kind of 'very local' monotonicity, i.e., pertaining to players of the same a priori union, which we formalized earlier for a fixed game might be expected to hold for power of a given player in the same a priori union in two different games:

Definition 7 A power index $g: \mathcal{W}^{\mathcal{P}}(N) \rightarrow \mathbb{R}^{n}$ is globally monotonic within unions if for every two weighted majority games with the same coalition structure and quota, i.e., $(N, v, P)$ and $\left(N, v^{\prime}, P\right) \in \mathcal{W}^{\mathcal{P}}(N)$ with $(N, v)=\left[q ; w_{1}, w_{2}, \ldots, w_{n}\right]$ and $\left(N, v^{\prime}\right)=\left[q ; w_{1}^{\prime}, w_{2}^{\prime}, \ldots, w_{n}^{\prime}\right] \in \mathcal{W}(N)$ and $P=\left\{P_{1}, P_{2}, \ldots, P_{m}\right\} \in \mathcal{P}(N)$,

$$
g_{i}(N, v, P) \geq g_{i}\left(N, v^{\prime}, P\right)
$$

holds for every player $i \in P_{k}$ such that $w_{i}>w_{i}^{\prime}, w_{j} \leq w_{j}^{\prime}$ for all $j \in P_{k} \backslash i$, and $w_{j}=w_{j}^{\prime}$ for all $j \in P_{l}, l \neq k$. 
This property is weaker than conventional global monotonicity because it restricts player $i$ 's power in $(N, v, P)$ and $\left(N, v^{\prime}, P\right)$ only when weights for all players outside the a priori union $P_{k}$ which contains $i$ are identical in both games. If a power index satisfies global monotonicity then it also satisfies global monotonicity within unions. In turn, global monotonicity within unions guarantees only that no violation of global monotonicity occurs for games with the coalition structure $P=P^{N}$. We have

Proposition 3 The BO, SCB and $O W$ indices satisfy global monotonicity within unions.

Proof. Consider two weighted majority games $(N, v, P)$ and $\left(N, v^{\prime}, P\right)$ with $(N, v)=\left[q ; w_{1}, w_{2}, \ldots, w_{n}\right]$ and $\left(N, v^{\prime}\right)=\left[q ; w_{1}^{\prime}, w_{2}^{\prime}, \ldots, w_{n}^{\prime}\right]$ satisfying $w_{i}>w_{i}^{\prime}, w_{j} \leq w_{j}^{\prime}$ for all $j \in P_{k} \backslash i$, and $w_{j}=w_{j}^{\prime}$ for all $j \in P_{l}, l \neq k$, for some player $i \in P_{k}$. Now consider an arbitrary coalition $S \subsetneq N$ which does not contain player $i$. We immediately have

$$
\sum_{j \in S} w_{j}^{\prime} \geq \sum_{j \in S} w_{j}
$$

And from $\sum_{j} w_{j}=\sum_{j} w_{j}^{\prime}=1$ we get

$$
w_{i}-w_{i}^{\prime}=\sum_{j \neq i}\left(w_{j}^{\prime}-w_{j}\right) \geq \sum_{j \in S}\left(w_{j}^{\prime}-w_{j}\right)
$$

which implies

$$
\sum_{j \in S} w_{j}+w_{i} \geq \sum_{j \in S} w_{j}^{\prime}+w_{i}^{\prime}
$$

Recalling that $v(S)=0 \Longleftrightarrow \sum_{j \in S} w_{j}<q$ and $v(S)=1 \Longleftrightarrow \sum_{j \in S} w_{j} \geq q$, Eq. (3) implies

$$
v^{\prime}(S)=0 \Longrightarrow v(S)=0
$$

and Eq. (4) implies

$$
v^{\prime}(S \cup i)=1 \Longrightarrow v(S \cup i)=1 .
$$

So $i$ 's marginal contribution to any coalition is weakly greater in $(N, v)$ than in $\left(N, v^{\prime}\right)$. This is true in particular for all coalitions $Q_{R} \cup T$ considered in Eq. (2), and so the claim follows from observing that the respective coefficients $p_{R, T}^{i}$ are unaffected by weight changes.

It turns out that the link between global and local monotonicity via symmetry that exists for standard power indices (Turnovec, 1998) extends to the respective 'within unions'-notions of monotonicity for coalitional power indices:

Proposition 4 If a power index $g: \mathcal{W}^{\mathcal{P}}(N) \rightarrow \mathbb{R}^{n}$ is globally monotonic within unions and symmetric within unions, then it is also locally monotonic within unions.

Proof. Let $g: \mathcal{W}^{\mathcal{P}}(N) \rightarrow \mathbb{R}^{n}$ satisfy global monotonicity within unions and symmetry within unions. Suppose $g$ violates local monotonicity within unions. Then there exists a game $(N, v, P) \in$ 
$\mathcal{W}^{\mathcal{P}}(N)$, where $(N, v)=\left[q ; w_{1}, w_{2}, \ldots, w_{n}\right]$ and $P=\left\{P_{1}, P_{2}, \ldots, P_{m}\right\}$, such that there exist two players $i, j \in P_{k}, k \in M=\{1, \ldots, m\}$ with $w_{i}>w_{j}$ and $g_{i}(N, v, P)<g_{j}(N, v, P)$.

Now consider the game $\left(N, v^{\prime}, P\right) \in \mathcal{W}^{\mathcal{P}}(N)$ with $\left(N, v^{\prime}\right)=\left[q ; w_{1}^{\prime}, w_{2}^{\prime}, \ldots, w_{n}^{\prime}\right]$ defined by $w_{i}^{\prime}=$ $w_{i}-\left(w_{i}-w_{j}\right) / 2, w_{j}^{\prime}=w_{j}+\left(w_{i}-w_{j}\right) / 2$, and $w_{h}=w_{h}^{\prime}$ for all $h \neq i, j$. Global monotonicity within unions implies

$$
\begin{gathered}
g_{i}(N, v, P) \geq g_{i}\left(N, v^{\prime}, P\right) \\
g_{j}\left(N, v^{\prime}, P\right) \geq g_{j}(N, v, P) .
\end{gathered}
$$

And, given that $w_{i}^{\prime}=w_{j}^{\prime}$, symmetry within unions implies

$$
g_{i}\left(N, v^{\prime}, P\right)=g_{j}\left(N, v^{\prime}, P\right)
$$

So $g_{i}(N, v, P) \geq g_{j}(N, v, P)$, establishing a contradiction.

\subsection{Global monotonicity between unions}

It remains to relate the aggregate power of a priori unions to their aggregate voting weights in different but (in order to be meaningful) closely related games:

Definition 8 A power index $g: \mathcal{W}^{\mathcal{P}}(N) \rightarrow \mathbb{R}^{n}$ is globally monotonic between unions if for every two weighted majority games with the same coalition structure and quota, i.e., $(N, v, P)$ and $\left(N, v^{\prime}, P\right) \in \mathcal{W}^{\mathcal{P}}(N)$ with $(N, v)=\left[q ; w_{1}, w_{2}, \ldots, w_{n}\right]$ and $\left(N, v^{\prime}\right)=\left[q ; w_{1}^{\prime}, w_{2}^{\prime}, \ldots, w_{n}^{\prime}\right] \in \mathcal{W}(N)$ and $P=\left\{P_{1}, P_{2}, \ldots, P_{m}\right\} \in \mathcal{P}(N)$,

$$
\sum_{i \in P_{k}} g_{i}(N, v, P) \geq \sum_{i \in P_{k}} g_{i}\left(N, v^{\prime}, P\right)
$$

holds for every union $P_{k} \in P$ such that $w\left(P_{k}\right)=\sum_{i \in P_{k}} w_{i}>w^{\prime}\left(P_{k}\right)=\sum_{i \in P_{k}} w_{i}^{\prime}$ and $w\left(P_{l}\right) \leq$ $w^{\prime}\left(P_{l}\right)$ for all $l \neq k$.

The lack of local monotonicity between unions, identified earlier, already suggests that the BO index also violates global monotonicity between unions. For example, the games $(N, v, P)=$ $\left[\frac{17}{22} ; \frac{6}{22}, \frac{16}{22}, 0,0\right]$ and $\left(N, v^{\prime}, P\right)=\left[\frac{17}{22} ; \frac{7}{22}, \frac{5}{22}, \frac{5}{22}, \frac{5}{22}\right]$ with $P=\{\{1\},\{2,3,4\}\}$ satisfy

$$
\sum_{i \in\{2,3,4\}} w_{i}=\frac{16}{22}>\sum_{i \in\{2,3,4\}} w_{i}^{\prime}=\frac{15}{22} \text { and } \sum_{i \in\{1\}} w_{i}=\frac{6}{22}<\sum_{i \in\{1\}} w_{i}^{\prime}=\frac{7}{22}
$$

but one obtains

$$
\sum_{i \in\{2,3,4\}} g_{i}^{B O}(N, v, P)=\frac{1}{2}<\sum_{i \in\{2,3,4\}} g_{i}^{B O}\left(N, v^{\prime}, P\right)=\frac{3}{4} .
$$

A globally monotonic coalitional power index is also globally monotonic between unions; in contrast, global monotonicity between unions only guarantees power vectors in line with unconditional global monotonicity for games with coalition structure $P=P^{n}$. 
As in the case of monotonicity within unions, the global version of monotonicity between unions implies the respective local property provided that the index under consideration satisfies an additional symmetry condition:

Proposition 5 If a power index $g: \mathcal{W}^{\mathcal{P}}(N) \rightarrow \mathbb{R}^{n}$ is globally monotonic between unions and symmetric between unions, then it is also locally monotonic between unions.

Proof. Let $g$ satisfy global monotonicity between unions and symmetry between unions. Suppose that $g$ violates local monotonicity between unions. Then there exists a game $(N, v, P) \in \mathcal{W}^{\mathcal{P}}(N)$, where $(N, v)=\left[q ; w_{1}, w_{2}, \ldots, w_{n}\right]$ and $P=\left\{P_{1}, P_{2}, \ldots, P_{m}\right\}$, such that there exist two a priori unions $P_{k}, P_{l} \in P$ with $w\left(P_{k}\right)>w\left(P_{l}\right)$ and $\sum_{i \in P_{k}} g_{i}(N, v, P)<\sum_{i \in P_{l}} g_{i}(N, v, P)$.

Now consider the game $\left(N, v^{\prime}, P\right) \in \mathcal{W}^{\mathcal{P}}(N)$ with $\left(N, v^{\prime}\right)=\left[q ; w_{1}^{\prime}, w_{2}^{\prime}, \ldots, w_{n}^{\prime}\right]$ defined by

$$
w_{j}^{\prime}= \begin{cases}\left(\sum_{i \in P_{k}} w_{i}-\frac{1}{2}\left(\sum_{i \in P_{k}} w_{i}-\sum_{i \in P_{l}} w_{i}\right)\right) /\left|P_{k}\right| & \text { if } j \in P_{k}, \\ \left(\sum_{i \in P_{l}} w_{i}+\frac{1}{2}\left(\sum_{i \in P_{k}} w_{i}-\sum_{i \in P_{l}} w_{i}\right)\right) /\left|P_{l}\right| & \text { if } j \in P_{l}, \\ w_{j} & \text { if } j \notin P_{k} \cup P_{l}\end{cases}
$$

Global monotonicity between unions implies

$$
\begin{aligned}
& \sum_{i \in P_{k}} g_{i}(N, v, P) \geq \sum_{i \in P_{k}} g_{i}\left(N, v^{\prime}, P\right) \\
& \sum_{i \in P_{l}} g_{i}\left(N, v^{\prime}, P\right) \geq \sum_{i \in P_{l}} g_{i}(N, v, P) .
\end{aligned}
$$

And, given that $\sum_{i \in P_{k}} w_{i}^{\prime}=\sum_{i \in P_{l}} w_{i}^{\prime}$, symmetry between unions implies

$$
\sum_{i \in P_{k}} g_{i}\left(N, v^{\prime}, P\right)=\sum_{i \in P_{l}} g_{i}\left(N, v^{\prime}, P\right) .
$$

So $\sum_{i \in P_{k}} g_{i}(N, v, P) \geq \sum_{i \in P_{l}} g_{i}(N, v, P)$, establishing a contradiction.

As for the local analogue, it is possible to relate global monotonicity between unions of a coalitional power index to conventional global monotonicity of an underlying standard index:

Proposition 6 If $g: \mathcal{W}^{\mathcal{P}}(N) \rightarrow \mathbb{R}^{n}$ is a coalitional extension of $f: \mathcal{W}(N) \rightarrow \mathbb{R}^{n}$ and satisfies the quotient game property, then global monotonicity of $f$ implies global monotonicity between unions of $g$.

Proof. Let $g$ be a coalitional extension of $f$ that satisfies the quotient game property and consider any arbitrary game $(N, v, P)$, where $(N, v)=\left[q ; w_{1}, w_{2}, \ldots, w_{n}\right]$ and $P=\left\{P_{1}, P_{2}, \ldots, P_{m}\right\} \in$ $\mathcal{P}(N)$. The quotient game property and the fact that $g$ extends $f$ imply

$$
\sum_{i \in P_{k}} g_{i}(N, v, P)=g_{k}\left(M, v^{P}, P^{m}\right)=f_{k}\left(M, v^{P}\right)
$$




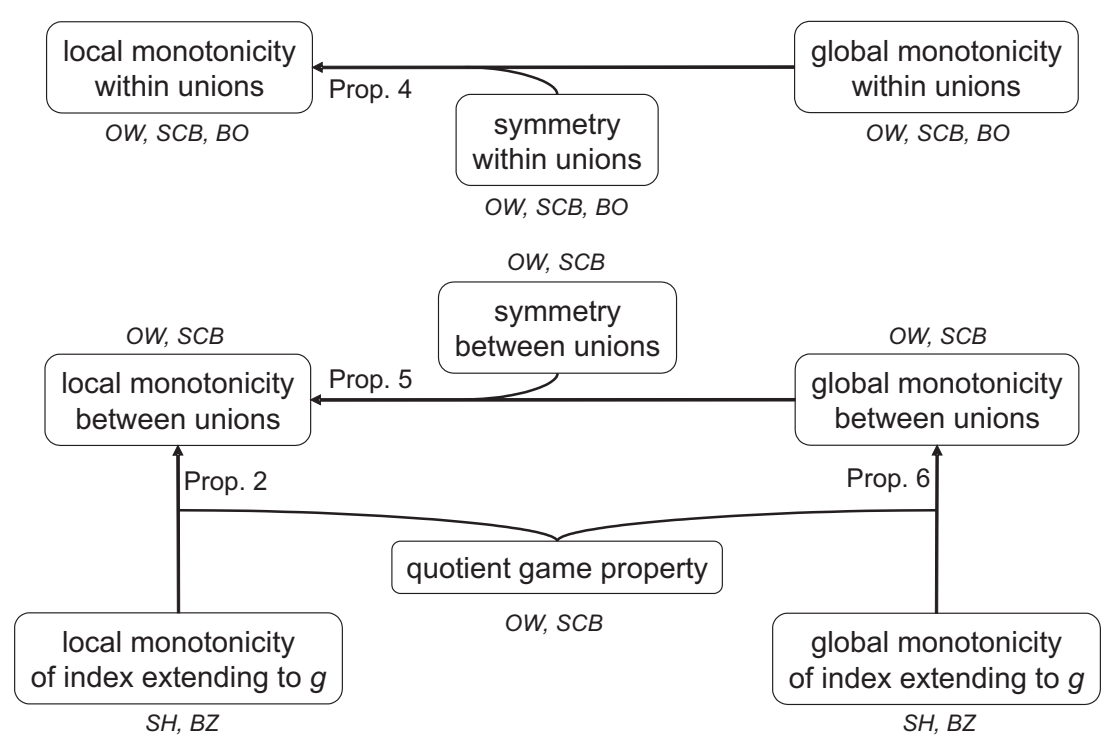

Figure 1: Relationship between monotonicity concepts

for each $P_{k} \in P$. For any game $\left(N, v^{\prime}, P\right)$ with the same coalition structure and quota, i.e., $\left(N, v^{\prime}\right)=\left[q ; w_{1}^{\prime}, w_{2}^{\prime}, \ldots, w_{n}^{\prime}\right] \in \mathcal{W}(N)$, one analogously has

$$
\sum_{i \in P_{k}} g_{i}\left(N, v^{\prime}, P\right)=g_{k}\left(M, v^{P}, P^{m}\right)=f_{k}\left(M,\left(v^{\prime}\right)^{P}\right)
$$

So

$$
\sum_{i \in P_{k}} g_{i}(N, v, P) \geq \sum_{i \in P_{k}} g_{i}\left(N, v^{\prime}, P\right)
$$

if and only if

$$
f_{k}\left(M, v^{P}\right) \geq f_{k}\left(M,\left(v^{\prime}\right)^{P}\right) .
$$

The latter and hence the former must be true given $w\left(P_{k}\right)>w^{\prime}\left(P_{k}\right)$ and $w\left(P_{l}\right) \leq w^{\prime}\left(P_{l}\right)$ for all $l \neq k$ whenever $f$ is globally monotonic.

Corollary 2 The $S C B$ and $O W$ indices are globally monotonic between unions.

\section{Concluding Remarks}

Coalitional power indices which take a priori unions into account fundamentally differ from standard power indices as they introduce an asymmetry among the players on top of the one created by different voting weights. Both types of asymmetry interact in a way that makes conventional notions of monotonicity - being merely a reflection of weight asymmetry - rather meaningless.

This paper adapted the conventional local and global monotonicity notions to take account of a priori unions. More precisely, we restricted the respective order requirement to player positions 
which remain meaningfully comparable in just the weight dimension after introduction of a nondegenerate coalition structure. This amounted to a power comparison (a) of players within a given union and (b) of the unions as actors themselves. The relationships identified in this paper between new and old concepts are illustrated in Figure $1 .^{8}$ Other useful monotonicity notions might be defined (e.g., relating players of distinct but still in some sense 'comparable' unions); we believe to have singled out the four most relevant ones.

It turned out that only the Owen index (Owen, 1977) and the Symmetric Coalitional Banzhaf index (Alonso-Meijide and Fiestras-Janeiro, 2002) generally exhibit weak monotonicity of power in weight within and between unions, at the local and at the global level. At first sight the BanzhafOwen index (Owen, 1982) might look like a more compelling coalitional extension of the standard Banzhaf index than the Symmetric Coalitional Banzhaf index: it imposes the equiprobability assumption of the Banzhaf index at both the within-unions and between-unions levels, while the Symmetric Coalitional Banzhaf index mixes assumptions of the Banzhaf index (between-unions level) and the Shapley-Shubik index (within-unions level). However, the seemingly more plausible Banzhaf-Owen index fails to be locally or globally monotonic between unions. We take this as an indication that, in fact, the Symmetric Coalitional Banzhaf index could be a more suitable indicator of power in settings involving a priori unions (e.g., formation of a government coalition). At least this is true when - in a specific context - a power indication is deemed plausible only when unions with given total weight are assigned at least as much power as unions with smaller total weight.

So just as standard notions of monotonicity help to discriminate between traditional power indices, the concepts defined in this paper can be applied to discriminate between coalitional power indices. Although the axiomatizations of traditional power indices suggest that this may be an unattainable goal (because none of them invokes monotonicity directly), it would be highly desirable to actually characterize rather than only 'stress test' coalitional power indices based on the proposed (or some other) monotonicity notions. In any case, the multitude of indices proposed for games without a priori unions allows for an even bigger number of coalitional power indices. So there is ample scope for applying the monotonicity concepts proposed here in order to quickly assess whether a particular combination is promising or not. It also seems worthwhile to investigate monotonicity properties relating not to the weight vector but rather the set of a priori unions. One might, e.g., want to require that a coalitional power index assigns no less than the sum of the power of two unions to the unit created by their merger. We leave this for future research.

\footnotetext{
${ }^{8} \mathrm{It}$ is straightforward that all considered indices are symmetric within unions. However, only the OW and SCB indices are also symmetric between unions (see the example after Definition 5).
} 


\section{Acknowledgments}

Financial support by the Ministerio de Ciencia y Tecnología through Project BEC 2002-04102-C0202 and the Xunta de Galicia through Project PGIDT00PXI20703PN are gratefully acknowledged.

\section{References}

[1] J.M. Alonso-Meijide and M.G. Fiestras-Janeiro (2002). "Modification of the Banzhaf value for games with a coalition structure", Annals of Operations Research, 109, 213-227.

[2] J.M. Alonso-Meijide and C. Bowles (2005). "Generating functions for coalitional power indices: An application to the IMF", Annals of Operations Research, 137, 21-44.

[3] J.M. Alonso-Meijide, F. Carreras, and M.G. Fiestras-Janeiro (2005). "The multilinear extension and the symmetric coalition Banzhaf value", Theory and Decision, 59, 111-126.

[4] R. Amer, F. Carreras, and J.M. Giménez (2002). "The modified Banzhaf value for games with a coalition structure: An axiomatic characterization", Mathematical Social Sciences, 43, $45-54$.

[5] R.J. Aumann and J. Drèze (1974). "Cooperative games with coalition strutures", International Journal of Game Theory, 3, 217-237.

[6] J.F. Banzhaf III (1965). "Weighted voting does not work: A mathematical analysis", Rutgers Law Review, 19, 317-343.

[7] F. Carreras and G. Owen (1988). "Evaluation of the Catalonian Parliament, 1980-1994", Mathematical Social Sciences, 15, 87-92.

[8] F. Carreras and A. Magaña (1994). "The multilinear extension and the modified BanzhafColeman index", Mathematical Social Sciences, 28, 215-222.

[9] J. Coleman (1971). "Control of collectivities and the power of a collectivity to act", in B. Lieberman (Ed.), Social Choice, New York: Gordon and Breach, 269-300.

[10] D.S. Felsenthal and M. Machover (2006). "Voting power measurement: A story of misreinvention", Social Choice and Welfare, 25, 485-506.

[11] G. Hamiache (1999). "A new axiomatization of the Owen value for games with coalition structures", Mathematical Social Sciences 37, 281-305.

[12] M.J. Holler and S. Napel (2004a). "Local monotonicity of power: Axiom or just a property?", Quality and Quantity, 38, 637-647. 
[13] M.J. Holler and S. Napel (2004b). "Monotonicity of power and power measures", Theory and Decision, 56, 93-111.

[14] G. Owen (1977). "Values of games with a priori unions", in R. Henn and O. Moeschlin (Eds.), Mathematical Economics and Game Theory. Berlin: Springer-Verlag, pp. 76-88.

[15] G. Owen (1982). "Modification of the Banzhaf-Coleman index for games with a priori unions", in M.J. Holler (Ed.), Power, Voting and Voting Power, Würzburg: Physica-Verlag, pp. 232 238.

[16] G. Owen and E. Winter (1992). "Multilinear extensions and the coalitional value", Games and Economic Behavior, 4, 582-587.

[17] L.S. Penrose (1946). "The elementary statistics of majority voting", Journal of the Royal Statistical Society, 109, 53-57.

[18] L.S. Shapley and M. Shubik (1954). "A method for evaluating the distribution of power in a committee system", American Political Science Review, 48, 787-792.

[19] A.D. Taylor and W.S. Zwicker (1999). Simple Games, Princeton, NJ: Princeton University Press.

[20] F. Turnovec (1998). "Monotonicity and power indices", in T.J. Stewart and R.C. van den Honert (Eds.), Trends in Multicriteria Decision Making, Lectures Notes in Economics and Mathematical Systems 465, Berlin: Springer-Verlag, pp. 199-214.

[21] M. Vázquez Brage, A. van den Nouweland, and I. García-Jurado (1997). "Owen's coalitional value and aircraft landing fees", Mathematical Social Sciences, 34, 273-286.

[22] E. Winter (1992). "The consistency and potential for values of games with coalition structure", Games and Economic Behavior, 4, 132-144. 


\section{Seit 1999 sind erschienen:}

NR. 103: M. J. HolLER, On the Regulation of Telecommunications Markets, March 1999.

NR. 104: Y. AltunBas, S. P. Chakravaty AND F. STEFFen, Proportional Representation in the National Assembly for Wales, March 1999.

NR. 105: M. J. HolLER AND B.-A. WICKSTRÖM, The Use of Scandals in the Progress of Society, May 1999.

NR. 106: R. ONO, Limit Property of a Multi-Choice Value: Comparison with the Fuzzy Value, June 1999.

NR. 107: M. J. Holler, R. ONO, AND F. STEFFEN, Constrained Monotonicity and the Measurement of Power, October 1999.

NR. 108: M. J. HoLLER, Fairness, Power and Their Relationship, November 1999.

NR. 109: F. STEFFEN, When is A Priori Voting Power really A Priori?, November 1999.

NR. 110: A. PomPERMAIER, Downsizing, X-efficiency and Social Efficiency, February 2000.

NR. 111: I. LiNDNER AND H. STRULIK, Property Rights and Growth, February 2000.

NR. 112: A. POMPERMAIER, Imperfect Competition and Firm Efficiency: A General Equilibrium Approach, April 2000.

NR. 113: M. J. HoLLER, Telecommunications Regulation and Economic Development, May 2000.

NR. 114: M. J. Holler AND V. KanniAinen, Compatibility Effects of Set-up Costs in the Organization of Service Systems, May 2000.

NR. 115: E. SCHOLING, Messung der Bevölkerungsgesundheit in Entwicklungsländern auf der Grundlage eines MIMIC-Modells, May 2000.

NR. 116: C. MeNZEL, Collusion in Auctions: A Rotating-Bid Mechanism, July 2000.

NR. 117: C. Hillinger, Consistent Aggregation and Chaining of Price and Quantity

Measures, August 2000.

NR. 118: M. J. HOLLER AND S. NAPEL, On Interpersonnel Comparison of Value, October 2000.

NR. 119: M. BrahAM AND F. STEFFEN, Voting Power in Games with Abstentions, July 2001.

NR. 120: M. BRAHAM AND F. STEFFEN, Notes on Power in Hierarchical Structures, July 2001.

NR. 121: I. LINDNER, Probabilistic Characterization of Power Indices with Abstentions, July 2001.

NR. 122: M. BRAHAM AND F. STEFFEN, Voting Rules in Insolvency Law: A Simple Game Theoretic Approach, December 2001.

NR. 123: M.J. HoLlER, Classical, Modern, and New Game Theory, December 2001.

NR. 124: I. LindNER AND M. MACHOVER, L.S. Penrose's Limit Theorem: Proof of a Special

Case, April 2002. 
NR. 125: I. LiNDNER AND H. STRULIK, Distributive Politics and Economic Growth: The Markovian Stackelberg Solution, April 2002.

NR. 126: M.J. HOLLER AND S. NAPEL, A Pragmatic Interpretation of Ken Binmore's Theory of Justice, October 2002.

NR. 127: M.J. Holler AND G. OwEN, Present and future of Power Measures, December 2002.

NR. 128: M.J. HolLER AND P. SKOTT, The Importance of Setting the Agenda, June 2003.

NR. 129: M.J. HOLLER AND I.LINDNER, Mediation as signal, June 2003.

NR. 130: M.Braham AND M.J. Holler, The Impossibility of a Preference-based Power Index, July 2003.

NR. 131: F. BOLLE AND M. BRAHAM, A Difficulty with Oats: On trust, trustwortiness, and signalling, July 2003.

NR. 132: M.J. HOLLER, The Artist as a Secret Agent: Liberalism Against Populism, August 2003.

NR. 133: W.GÜTH AND S.NAPEL, Inequality Aversion in a Variety of Games: An Indirect Evolutionary Analysis, November 2003.

NR. 134: E. SCHOLING, Zur ökonomischen Analyse der Kinderarbeit, April 2004.

NR. 135: M. BRAHAM, Freedom, power, and success: A game-theoretic perspective, May 2004.

NR. 136: M.J. HOLLER AND H. NURMI, Power, Outcomes and Preferences, September 2004.

NR. 137: M.J. HOLLER, How to Sell Power Indices, September 2004.

NR. 138: M. BRAHAM AND F. STEFFEN, The Chairman's Paradox Revisited, October 2004.

NR. 139: M.J. HOLLER, George Orwell and His Cold Wars: Truth and Politics, January 2005.

NR. 140: R. HARADAU AND S. NAPEL, Holler-Packel Value and Index - A New

Characterization, January 2005.

NR. 141: D. MOOKHERJEE AND S. NAPEL, Intergenerational Mobility and Macroeconomic History Dependence, April 2005.

NR. 142: M.J. HOLLER, Freedon of Choice, Power, and the Responsibility of Decision Makers, September 2005.

NR. 143: M.J. HOLLER AND S. NAPEL, Democratic Decision Procedures and Stability with Reference to the European Union, March 2006.

NR. 144: M.J. HOLLER AND H. NURMI, Measurement of Power, Probabilities, and Alternative Models of Man, March 2006.

NR. 145: J.M. AlONSO-MEIJIDE, C. BOWLES, M.J. HOLLER AND S. NAPEL, Monotonicity of power in games with a priori unions, May 2006. 